\title{
Biological aspects of appendix pathology: irregular distribution of myenteric ganglia in human appendiceal wall
}

\author{
Kubikova E, Sivakova I, Perzelova A, El Falougy H \\ Department of Anatomy, Faculty of Medicine, Commenius University, Bratislava, Slovakia. \\ anna.perzelova@fmed.uniba.sk
}

\begin{abstract}
OBJECTIVES: Although appendicitis is a common disease, basic questions about risk factors and its etiology remain unexplained.

BACKGROUND: An obstruction of the appendix lumen is usually considered to be the main cause of acute appendicitis. However, more studies are currently dealing with neuroimmune appendicitis.

METHODS: We studied samples of human appendices with the histological diagnosis of chronic appendicitis. Fixed cryosections of appendiceal walls were examined by immunofluorescence methods using neuronal antineurofilament antibody markers and beta III tubulin.

RESULTS: The immunostaining revealed an irregular distribution of myenteric ganglia in inflamed appendiceal walls and unexpected groups of large ganglia unequally distributed in the subserosal area. The comparative analysis of normal and inflamed appendix samples showed differences in the occurrence of myenteric ganglia in the subserosal area. They appeared more frequently on cryosections prepared from the inflamed appendiceal wall.

CONCLUSION: We propose that the high variability and irregular location of myenteric ganglia in the appendiceal wall are due to an alteration in the motility which results in flaccid appendix emptying. In addition, superficially located myenteric ganglia are exposed to abdominal irritation and may explain the chronic abdominal pain which is often considered to be a sign of chronic appendicitis (Fig. 2, Ref. 23). Text in PDF www.elis.sk. KEY WORDS: chronic appendicitis, chronic abdominal pain, myenteric ganglia, neurofilaments, beta III tubulin.
\end{abstract}

\section{Introduction}

Appendicitis may occur either as acute or chronic inflammation of the appendix vermiformis. Despite the diagnostic and therapeutic advancement in medicine, appendicitis remains a clinical emergency and is one of the common causes of acute abdominal pain. Still, the etiology of appendicitis remains unclear. Acute appendicitis is most common between 10 to 20 years of age but can occur at all ages $(1,2)$. Increased risks for appendicitis were described chiefly as low-fiber $\operatorname{diet}(3,4)$, infections due to edema causing obstruction of appendix lumen, and hereditary factors (5). Familial history of appendicitis is considered to be due to a particular positioning of the appendix, which predisposes it to infection (6). In animals, appendicitis has been described only in anthropoid apes (7) with characteristically narrow worm-shaped

Department of Anatomy, Faculty of Medicine, Commenius University, Bratislava, Slovakia

Address for correspondence: A. Perzelova, Department of Anatomy, Faculty of Medicine, Comenius University, Sasinkova 2, SK-813 72 Bratislava, Slovakia.

Phone: +421.2 .59357424$

Acknowledgements: We would like to thank Dr. MacLeod for critical reading of the manuscript and Mrs. Hillova, Mrs. Skopekova, Mrs. Galfyova and Mr. Miklos for technical assistance. This study was supported by VEGA grant No. 1/3439/06 and ITMS: 26240120023, co-financed by the European Regional Development. appendix. On the other hand, in many primates and mammals, the appendix is much more open and sack-shaped (8).

Recently, more attention has been paid to the appendix innervation in relation to appendix emptying. Normal gastrointestinal motility depends on the enteric nervous system and networks of interstitial cells of Cajal which generate the pacemaker signals $(9,10)$. Previously, we examined myenteric ganglia of apparently normal appendices using neuronal marker antibodies. The aim of this study was to demonstrate the differences in myenteric ganglia localization in the normal and inflamed appendiceal wall by immunohistochemical methods.

\section{Material and methods}

\section{Patients and samples}

Patients with the clinical diagnosis of appendicitis underwent appendectomy at Children's Faculty Hospital, Bratislava. Parts of 50 appendices were obtained for our studies. The histological diagnosis was performed by pathologists at Cytopathos, s.r.o. Bratislava. Experiments with human bioptic samples were approved by the Ethical Committee of UNB Bratislava. For this study, we chose appendices from five patients (15-18 years of age) with the histological diagnosis of chronic appendicitis. The appendix samples were cut along their longitudinal axes. Specimens for cryosections were embedded with OCT and cut into 10$\mu \mathrm{m}$ thick sections. 
Immunofluorescence staining

Cryosections were fixed in methanol-acetone (1:1) solution for $15 \mathrm{~min}$ at $-15{ }^{\circ} \mathrm{C}$ or with $4 \% \mathrm{p}$-formaldehyde for $15 \mathrm{~min}$ at room temperature. Myenteric ganglia were detected by indirect immunofluorescence staining using neuronal anti-neurofilament antibody marker (1 : 100 dilution, clone NF-01, Exbio, Prague) and two antibodies against beta III tubulin, monoclonal (1:100, TUJ 1, Abcam), and polyclonal sera developed in rabbit (1:100, Sigma). Fixed cryosections were incubated for 1 hour with primary antibodies and for 30 minutes with $1: 50$ diluted secondary antibodies (Sigma). Double labeling with NF-01 and polyclonal sera against beta III tubulin was performed with primary antibodies, and afterwards with appropriate mixtures of secondary antibodies for 1 hour and 30 minutes, respectively. The staining of cryosections fixed with $\mathrm{p}$-formaldehyde was preceded by cell membrane permeabilization with $0.5 \%$ Triton X-100 for 15 min. Nuclei were stained with Hoechst 33342 (5 $\mu \mathrm{g} / \mathrm{ml}$ in PBS, Sigma) $1 \mathrm{~min}$.

\section{Results}

We have studied myenteric ganglia by indirect and doubleimmunofluorescence methods using neuronal anti-neurofilament (expressed only in neuronal cells) and anti-III tubulin antibody markers. Monoclonal antibodies NF-01 recognize an epitope on heavy neurofilament protein $(210 \mathrm{kDa})$ of various species (11). Beta III tubulin was found in the central and peripheral nervous systems, and appears to be specific for neurons $(12,13)$. However, beta III tubulin was also found in human fetal astrocytes (14). By double labeling, we previously demonstrated a similar staining with both neuronal marker antibodies on non-inflamed appendix cryosections (15). In this study we found a similar co-expression of neurofilaments and beta III tubulin on inflamed appendiceal wall sections. In addition, the staining with neurofilament was brighter than that with beta III tubulin. This is because the majority of cryosections were examined by indirect immunofluorescene with neurofilament antibodies.

The indirect immunofluorescence showed strong staining of myenteric ganglia with neurofilaments (Fig. 1A, B) and beta III tubulin antibodies (Fig. 1C, D). They were of different sizes and shapes irregularly distributed mainly in the circular layer (Fig. 1B, D) or within both muscle layers (Fig. 1A, C). We observed single or cumulated large ganglia in the subserosal area (Fig. 2A, B, C, D). Subserosal myenteric ganglia were very unequally distributed in all inflamed appendiceal samples.

\section{Discussion}

Previously, we demonstrated an irregular distribution and atypical subserosal localization of myenteric ganglia in the normal human appendiceal wall. In this study, we examined bioptic samples from patients with the histological diagnosis of chronic appendicitis. To perform the comparative analysis of myenteric plexuses in normal and inflamed appendiceal walls we used the same methods and neuronal marker antibodies (15). Cryosections were stained with neurofiaments and beta III tubulin antibodies. Similarly to the normal appendiceal wall, the immunostaining revealed an irregular distribution of myenteric ganglia in inflamed appendices. However, some differences were found in the occurrence of myenteric ganglia in the subserosal area. In the normal appendix, small ganglia rarely appeared in the subserosa. On the other hand, in inflamed appendices, they occurred more frequently. It is remarkable that several myenteric ganglia of varying size and shape were cumulated in the subserosa. Single or cumulated subserosal ganglia appeared to be very unequally distributed in
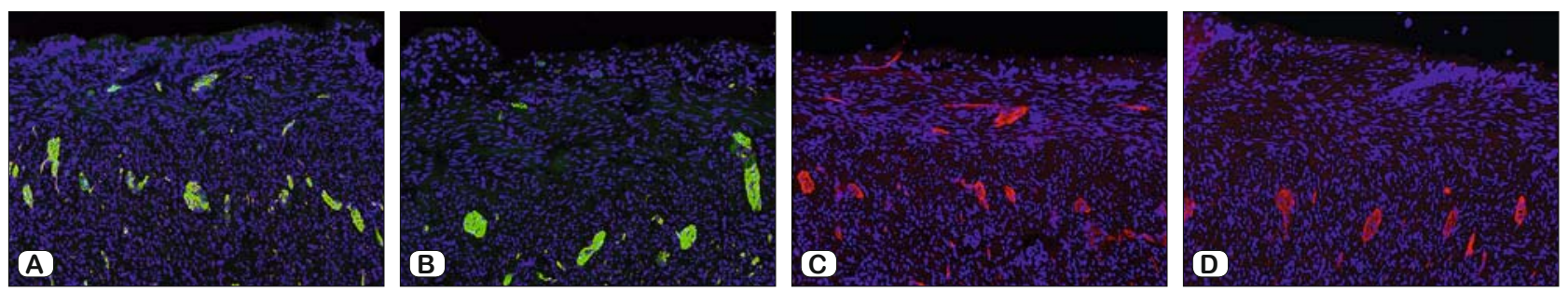

Fig. 1. Localization of myenteric ganglia in human appendiceal wall with the diagnosis of chronic appendicitis. Indirect immunofluorescence for neurofilaments (A, B) and beta III tubulin (C, D), stained longitudinal cryosections. Myenteric ganglia were distributed mainly within the circular layer (A, C) or within longitudinal and circular muscle layers (B, D). Nuclei stained with Hoechst. Scale $200 \mu \mathrm{m}$.
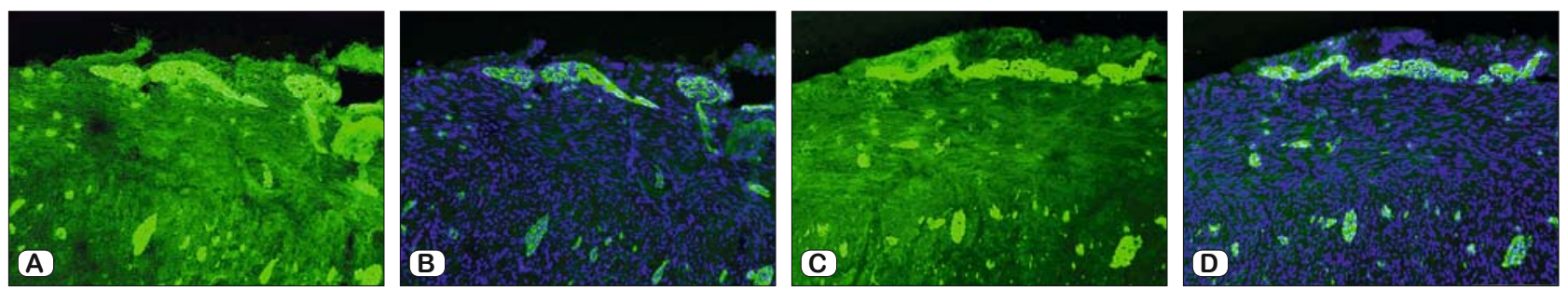

Fig. 2. Concentrated myenteric ganglia in the subserosal area of human appendix with diagnosed chronic appendicitis. Indirect immunofluorescence staining on longitudinal cryosections using antibodies against neurofilaments (A, B). Stained for neurofilaments and nuclei stained with Hoechst (B, D). Scale $200 \mu \mathrm{m}$. 


\section{$726-728$}

all inflamed appendices. The irregular distribution of myenteric ganglia in human appendix was previously described in some studies $(16,17)$. On the other hand, Hanani (18) revealed that in most cases, the innervation of the external muscles of the appendix consisted of three concentric networks of ganglia located between the circular and longitudinal muscle layers as well as within them.

The most important question about the human appendix is the poorly understood etiology of appendicitis in children and young adults. Obstruction of the appendix lumen is usually considered to be the main cause of acute appendicitis (19). However, recent studies measuring the pressure in the appendix perioperatively suggest that the obstuction is not an important causative agent of acute appendicitis, but might develop as a result of the inflamatory process (20). Other authors described no significant correlation between gangrenous/perforated appendicitis and the presence of a fecalith (21). Currently more studies are dealing with neuroimmune appendicitis or neuronal hypertrophy in acute appendicitis $(22,23)$.

These results confirm our lately advanced hypothesis that the irregular distribution of myenteric ganglia occurs due to an alteration in motility which results in flaccid appendix emptying and subsequent pathogenesis of human appendicitis (15). In this study, we revealed areas of subserosal large myenteric ganglia in an inflamed appendiceal wall. Thus, superficially located myenteric ganglia are exposed to abdominal irritation and may help explain the chronic abdominal pain which is often considered to be a sign of chronic appendicitis. We infer that the most important role in appendicitis etiology is the variability and irregular distribution of myenteric ganglia in the appendiceal wall, while the mentioned risks for appendicitis may only develop this disease.

\section{References}

1. Addis DG, Shaffer N, Fowler BS, Tauxe RV. The epidemiology of appendicitis and appendectomy in the United States. Am J Epidemiol 1990; 132: 910-925.

2. Anderson JE, Bickler S, Chang DC, Talamini MA. Examining a common disease with unknown etiology: Trends in epidemiology and surgical management of appendicitis in California, 1995-2009. World J Surg 2012; 36: 2787-2794

3. Burkitt DP. The aetiology of appendicitis. Br J Surg 1971; 58: 695-699.

4. Adamidis DI, Roma-Giannikou E, Karamolegou K, Tselalidou E, Constantopoulos A. Fiber intake and childhood appendicitis. Int J Food Sci Nutr 2000; 51 (3): 153-7.

5. Andersson N, Griffiths H, Murphy J, Roll J, Serenyi A, Swann I, Cockcroft A, Myers J, Leger AST. Is appendicitis familial? Brit Med J 1979; 2: 697-698.

6. Shperber Y, Halevy A, Oland J, Orda R. Familial retrocaecal appendicitis. J Royal Soc 1986; 79 (7): 405-406.
7. Scott GBD. The primate caecum and appendix vermiformis a comparative study. J Anat 1980; 131 (3): 549-563.

8. Smith HF, Fisher RE, Everett ML, Thomas AD, Bollinger RR, Parker W. Comparative anatomy and phylogenetic distribution of the mammalian cecal appendix. J Evol Biol 2009; 22 (10): 1984-1999.

9. Thuneberg L. Interstitial cells of Cajal : Intestinal pacemaker cells? Adv Anat Embryol Cell Biol 1982; 71: 1-130.

10. Čamborová P, Hubka P, Šulková I, Hulín I. The pacemaker aktivity of interstitial cells of cajal and elektrical activity. Physiol Res 2003; 52: $275-284$.

11. Lukas Z, Draber P, Bucek J, Draberova E, Viklický V, Dolezel S. Expression of phosphorylated high molecular weight neurofilament protein (NF-H) and vimentin in human developing dorsal root ganglia and spinal cord. Histochemistry 1993; 100: 495-502.

12. Menezes JRL, Luskin MB. Expresssion of neuron-specific tubulin defines a novel population in the proliferative layers of the developing telencephalon. J Neurosci 1994; 14: 5399-5416.

13. Ferreira A, Caceres A. Expression of the class III beta-tubulin isotype in developing neurons in culture. J Neurosci Res 1992; 32: 516-529.

14. Draberová E, Del Valle L, Gordon J, Marková V, Smejkalová B, Bertrand L, de Chadarévian JP, Agamanolis DP, Legido A, Khalili $\mathbf{K}$, Dráber P, Katsetos CD. Class III beta-tubulin is constitutively coexpressed with glial fibrillary acidic protein and nestin in midgestational human fetal astrocytes: implications for phenotypic identity. J Neuropathol Exp Neurol 2008; 67: 341-354.

15. Kubikova E, Sivakova I, Perzelova A. Atypical localization of myenteric ganglia in the human appendical wall: a comparative study with animal appendix. Biologia 2014; 69: 931-935.

16. Emery JL, Underwood J. The neurological junction between the appendix and ascending colon. Gut 1970; 11: 18-120.

17. Rajeswara RN, Kommuru H, Anuradha S, Swayam JS. Ganglion cells of the myenteric plexus in human vermiform appendix. J Med Sci Tech 2013; 3 (1).

18. Hanani M. Multiple myenteric networks in the human appendix. Auton Neurosci -Basic 2004; 110: 49-54.

19. Pieper R, Kager L, Tidefeldt U. Obstruction of appendix vermiformis causing acute appendicitis. Acta Chir Scand 1982; 148: 63-72.

20. Arnbjörnsson E, Bengmark S. Role of obstruction in the pathogenesis of acute appendicitis. Am J Surg 1984; 147 (3): 390-392.

21. Ramdass MJ, Sing QY, Milne D, Mooteeram J, Barrow S. Association between the appendix and the fecalit in adults. J Can Chir 2015; 58 (1): $10-14$.

22. Di Sebastiano P, Fink T, Mola F, Weihe E, Innocenti P, Friess H, Buchler M. Neuroimmune appendicitis. The Lancet 1999; 354: 461-466.

23. Xiong S, Puri P, Nemeth L, Brian S, Reen D. Neuronal Hypertrophy in acute appendicitis. Arch Pathol Lab Med 2000; 124: 1429-1433.

Received March 16, 2015. Accepted June 26, 2015. 